\title{
Prevalence and risk factors of postoperative delirium after spinal surgery: a meta- analysis
}

\author{
Hua Gao ${ }^{1 *}$, Hui-Juan Ma ${ }^{2}$, Ying-Jia Li ${ }^{2}, \mathrm{Ci} \mathrm{Yin}^{3}$ and Zheng Li
}

\begin{abstract}
Objective: Postoperative delirium (POD) was common after spinal surgery, but the main findings in previous studies remained conflicting. This current meta-analysis was aimed at exploring the prevalence and risk factors of POD after spinal surgery.

Methods: PubMed and Embase were searched from inception to June 2019. Studies which reported the prevalence and risk factors of POD after spinal surgery were included. STATA version 12.0 was employed to analyze the pooled data. Statistical heterogeneity across included studies was identified using the $P$ statistics.

Results: A total of 28 studies with 588,732 patients were included in the meta-analysis. The pooled prevalence of POD after spinal surgery was $0.85 \%(95 \% \mathrm{Cl}, 0.83-0.88 \%)$ with substantial heterogeneity $\left(I^{2}=97.3 \%\right)$. The central nervous system disorder (OR 4.73; 95\% $\mathrm{Cl}, 4.30-5.19)$ was a strong predictor for $\mathrm{POD}$, whereas age $(\mathrm{OR} 1.16 ; 95 \% \mathrm{Cl}$, $\left.1.05-2.47 ; P^{2}=99.2 \%\right)$ and blood loss $\left(\mathrm{OR} 1.10 ; 95 \% \mathrm{Cl}, 1.01-1.20 ; P^{2}=93.3 \%\right)$ were weaker predictors. The funnel plot and statistical tests suggested that there existed potential publication bias, but the trim and fill method indicated that the pooled prevalence basically kept stable after adding two "missing" studies.

Conclusions: The pooled POD after spinal surgery ranges from 0.83 to $0.88 \%$. The central nervous system disorder, age, and blood loss were potential risk factors for POD.
\end{abstract}

Keywords: Delirium, Prevalence, Risk factor, Spinal surgery, Meta-analysis

\section{Introduction}

Delirium, an acute state of confusion, is characterized as the distortion in consciousness and perception, decreased capacity of focusing one's attention, deteriorated cognitive functions, and disturbed sleep-wake cycles [1, 2]. Postoperative delirium (POD) is a common complication after any major surgical procedure, which predominantly occurs in elderly $[3,4]$. POD is associated with loss of independence, longer hospital stay, aggravated cognitive capacity, increased morbidity and mortality

\footnotetext{
*Correspondence: gao.hua@outlook.com

'Department of Outpatient, Lanzhou University Second Hospital, Lanzhou University Second Clinical Medical College, Lanzhou 730030, China Full list of author information is available at the end of the article
}

risk, and greater medical economic burden [5-7]. Unfortunately, the treatments for POD are full of challenges currently [3]. In general, identifying POD-associated risk factors is a potential useful way to understand the characteristics of POD, so it is essential to identify the potential perioperative risk factors which may help to establish effective strategies for prevention and treatment. In 2015, Shi et al. performed a meta-analysis to identify the POD-associated risk factors after spinal surgery [8]. However, the previous meta-analysis only included six studies, and thus, the reliability of its conclusion may be limited by the small sample size. Moreover, following the meta-analysis by Shi and coworkers, a large body of studies was performed to make 
a further exploration on the POD-associated risk factors after spinal surgery. Additionally, an accurate estimation of the POD incidence is also of much significance. On the one hand, in the intervention studies without a placebo group, a precise estimate of incidence is needed for comparison to determine whether the intervention could effectively prevent POD. The researchers could calculate the appropriate number of subjects needed for intervention studies based on the incidence [9]. On the other hand, an accurate estimate of incidence may help to identify some certain subgroups of patients, which would attract more attention of doctors to adjust interventions for the specific patient populations, and it meanwhile may guide researchers to establish the scientific inclusion of clinical trials, matching the interventional and control groups well in terms of POD-associated baseline risk variables. Nevertheless, currently, the reported prevalences of POD vary too widely following spinal surgery with inconsistency.

Therefore, in this meta-analysis, we combined the currently available studies to systematically examine the prevalence and risk factors of POD following spinal surgery.

\section{Methods}

This meta-analysis was performed according to the guideline of the Meta-analysis of Observational Studies in Epidemiology checklist and the Cochrane Handbook [10]. Two reviewers separately performed selection criteria, data extraction, quality assessment, and statistical analysis, with inconsistence resolves by a third reviewer.

\section{Search strategy and selection criteria}

A systematic literature search was performed in PubMed and Embase for studies published before June 10, 2019. The search terms included delirium, risk factor, spinal surgery, and their variants. Additionally, the reference lists of the eligible studies and relevant reviews were carefully screened to identify any potential inclusion. All eligible observational studies, which reported the incidence of delirium after spinal surgery or provided relevant information to calculate the incidence of POD, were assessed for inclusion in this meta-analysis. Studies enrolling fewer than 25 subjects, with overlapping patients, or without available data were excluded. When two or more studies included the overlapping populations, the one with the largest sample size and the longest duration was chosen for the current meta-analysis.

\section{Data extraction}

Two co-authors independently extracted relevant data by using a pre-determined Excel sheet. The items of data extraction included the first author, year of publication, country, years of survey, type of operation, study design, mean age, sample size, risk factor, the number of delirium, and delirium assessment methods. The primary outcome is the prevalence of POD after spinal surgery. Additionally, the odds ratios (ORs) with corresponding 95\% confidence intervals (CIs), which described the perioperative risk factors for POD after spinal surgery, were also extracted. Specially, we merely extracted individual risk factors which were assessed on multivariate or adjusted analysis in at least two studies.

\section{Quality assessment}

The quality of eligible studies was evaluated using the Newcastle-Ottawa Scale (NOS) score [11, 12]. This score system is established specifically for assessing the quality of observational studies, in which scores are assigned for three dimensions, including selection criteria of participants, comparability, exposure, and outcome. A maximum score of NOS is up to 9 , suggesting the highest quality.

\section{Data synthesis and analysis}

Stata SE12.0 (Stata Corp., College Station, TX, USA) was used to estimate the pooled prevalence of POD after spinal surgery. According to the Cochrane Handbook (9.5.4), the random-effects estimate and its confidence interval address the question "what is the average effect?" while the fixed-effect estimate and its confidence interval address the question "what is the best estimate of the effect?" When the results of pooled analysis based on random-effects model and fixed-effect model are very different from each other owing to substantial heterogeneity across included studies, the fixed-effect estimate and its confidence interval, but not the random-effects estimate may more truthfully reflect the pooled prevalence of POD in spine surgery. Besides, sensitivity analyses were performed by excluding one study at each step to further assess the influence of individual included studies on the overall synthesis analyses. If individual risk factors of interest were reported in two or more studies, the pooled OR estimates with 95\% CIs were calculated. Statistical heterogeneity across studies was evaluated using $I^{2}$ statistic $\left(I^{2}>50 \%\right.$ was regarded as substantial heterogeneity) [13, 14]. Subgroup analysis and meta-regression analysis for the primary outcome were used to detect the potential source of heterogeneity. The following categorical variables were analyzed in subgroup analyses: (1) region: Asia vs. Europe vs. North America; (2) year of the survey: before 2010 vs. after 2010 by the median splitting method; (3) sample size: $\leq$ 500 vs. $>500$ by the median splitting method; (4) type of operation: oncological spine surgery vs. non-oncological spinal surgery; (5) mean age of patients: $\leq 60$ vs. $>60$; (6) study design: database analysis vs. non-database related observational study; and (7) preoperative disease 
status: with preoperative cerebrovascular disorders vs. without preoperative cerebrovascular disorders. Continuous variables including age, year of publication, and NOS score were analyzed by meta-regression analysis. Publication bias was assessed using Begg and Egger's test and funnel plot $[15,16]$. If there was significant publication bias, the "trim and fill method" was used to determine whether it obviously affected the robustness of the synthesis analysis [17]. $P \leq 0.05$ was deemed to be statistically significant.

\section{Results}

\section{Study selection and characteristics}

A total of 576 articles were identified initially. After removing duplicate records and irrelative titles and abstracts, the full texts of 66 articles were further screened for eligibility. Finally, a total of 28 studies with 588,732 patients were included in this meta-analysis [18-45]. The flow diagram for the study selection was presented in Fig. 1. The included studies were published from 2006 to 2019. Among all eligible studies, two were performed in Europe, nine in North America, and the others in
Asia. The detailed characteristics of the included studies were shown in Table 1 . The NOS score of all the included studies ranged from 6 to 9 , suggesting the quality of included studies was relatively high for the current meta-analysis. The detailed NOS score of the included studies are shown in Table 2.

\section{Postoperative delirium after spine surgery}

All included studies reported POD after spinal surgery. The pooled prevalence of POD was $0.85 \%(95 \% \mathrm{CI}$, 0.0083-0.0088; $I^{2}=97.3 \%$; Fig. 2a) using fixed-effect model, but the pooled prevalence based on randomeffects model was $12 \%$ (95\%CI, 0.09-0.14; $I^{2}=97.3 \%$; Fig. 2b). According to the Cochrane Handbook, the fixed-effect estimate may more truthfully reflect the authentic pooled prevalence of POD in spine surgery when there existed a significant difference between the fixedeffect and random-effects estimates with substantial heterogeneity. Furthermore, we performed sensitivity analyses to explore the influence of individual included studies on the overall pooled effect. The results indicated that the pooled prevalence of POD basically remained

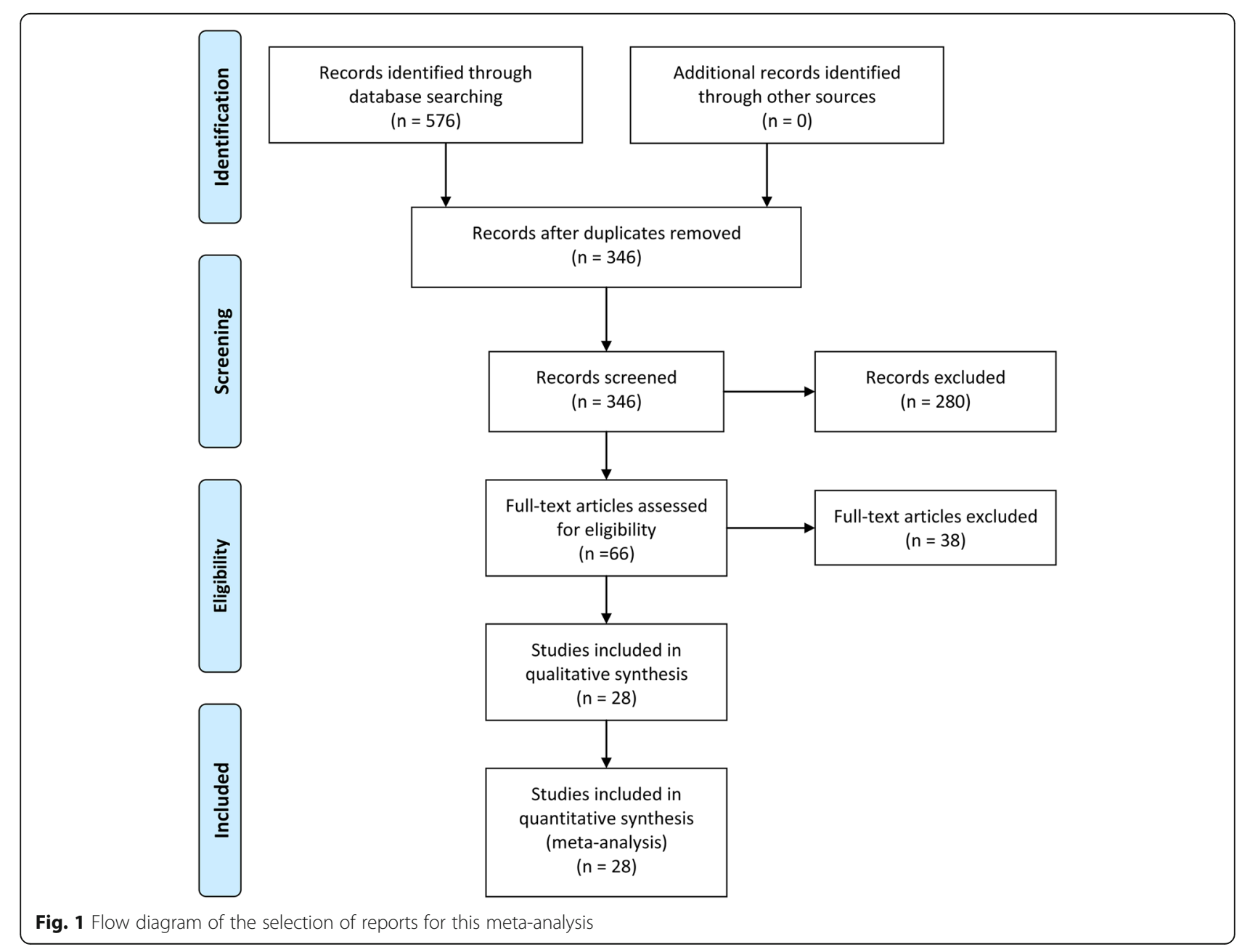


Table 1 Characteristics of included studies

\begin{tabular}{|c|c|c|c|c|c|c|c|c|}
\hline Author, year & Country & Year of survey & Type of operation & $\begin{array}{l}\text { Age } \\
(\text { mean, y) }\end{array}$ & Study design & $\begin{array}{l}\text { Sample } \\
\text { size }\end{array}$ & $\begin{array}{l}n \\
\text { (delirium) }\end{array}$ & Delirium assessment \\
\hline $\begin{array}{l}\text { Kawaguchi } \\
\text { et al. } 2006 \\
\text { [30] }\end{array}$ & Japan & $2000-2002$ & Mixed spine surgery & 59.2 & $\begin{array}{l}\text { Retrospective } \\
\text { cohort study }\end{array}$ & 341 & 13 & CAM \\
\hline $\begin{array}{l}\text { Cho et al. } \\
2007 \text { [21] }\end{array}$ & Korea & NA & $\begin{array}{l}\text { Degenerative lumbar } \\
\text { scoliosis surgery }\end{array}$ & 66.6 & $\begin{array}{l}\text { Retrospective } \\
\text { cohort study }\end{array}$ & 47 & 2 & NA \\
\hline $\begin{array}{l}\text { Gao et al. } \\
2008 \text { [27] }\end{array}$ & China & $\begin{array}{l}\text { (May- } \\
\text { November) } \\
2007\end{array}$ & Mixed spine surgery & 48.2 & Cross-sectional & 549 & 18 & DOS \\
\hline $\begin{array}{l}\text { Ushida et al. } \\
2009[45]\end{array}$ & Japan & 2003-2007 & Cervical surgery & 69.8 & $\begin{array}{l}\text { Retrospective } \\
\text { cohort study }\end{array}$ & 122 & 26 & DOS \\
\hline $\begin{array}{l}\text { Lee and Park } \\
2010[36]\end{array}$ & Korea & 2000-2007 & $\begin{array}{l}\text { Degenerative lumbar } \\
\text { disease }\end{array}$ & 73.5 & $\begin{array}{l}\text { Retrospective } \\
\text { cohort study }\end{array}$ & 217 & 11 & CAM \\
\hline $\begin{array}{l}\text { Kelly et al. } \\
2012 \text { [31] }\end{array}$ & Canada & 2009-2010 & $\begin{array}{l}\text { Degenerative } \\
\text { spondylolisthesis } \\
\text { surgery }\end{array}$ & 66.08 & Cross-sectional & 92 & 5 & NA \\
\hline $\begin{array}{l}\text { Li et al. } \\
2012[37]\end{array}$ & China & 2007-2011 & Mixed spine surgery & 75.3 & $\begin{array}{l}\text { Retrospective } \\
\text { cohort study }\end{array}$ & 1216 & 116 & CAM \\
\hline $\begin{array}{l}\text { Bollen et al. } \\
2013 \text { [19] }\end{array}$ & $\begin{array}{l}\text { The } \\
\text { Netherlands }\end{array}$ & $2001-2010$ & $\begin{array}{l}\text { Spinal epidural } \\
\text { metastases surgery }\end{array}$ & 59 & Cross-sectional & 106 & 3 & NA \\
\hline $\begin{array}{l}\text { Fineberg et al. } \\
2013 \text { [26] }\end{array}$ & $\begin{array}{l}\text { The United } \\
\text { States }\end{array}$ & 2002-2009 & $\begin{array}{l}\text { Lumbar } \\
\text { decompression and } \\
\text { fusion surgery }\end{array}$ & 55.1 & $\begin{array}{l}\text { Retrospective } \\
\text { database study }\end{array}$ & 578,457 & 4857 & ICD-9-CM \\
\hline $\begin{array}{l}\text { Dea et al. } \\
2014 \text { [23] }\end{array}$ & $\begin{array}{l}\text { The } \\
\text { Netherlands }\end{array}$ & 2009-2012 & $\begin{array}{l}\text { Oncological spine } \\
\text { surgery }\end{array}$ & 61.9 & Cross-sectional & 101 & 21 & NA \\
\hline $\begin{array}{l}\text { Seo et al. } \\
2014[42]\end{array}$ & Korea & 2012-2013 & Mixed spine surgery & 70.1 & $\begin{array}{l}\text { Case-control } \\
\text { study }\end{array}$ & 70 & 17 & DSM-5 \\
\hline $\begin{array}{l}\text { Glennie et al. } \\
2015 \text { [28] }\end{array}$ & Canada & 2009-2013 & $\begin{array}{l}\text { Thoracic and lumbar } \\
\text { spine fracture surgery }\end{array}$ & 44.3 & $\begin{array}{l}\text { Case-control } \\
\text { study }\end{array}$ & 276 & 38 & NA \\
\hline $\begin{array}{l}\text { Brown et al. } \\
2016[20]\end{array}$ & $\begin{array}{l}\text { The United } \\
\text { States }\end{array}$ & 2012-2014 & Mixed spine surgery & 74 & $\begin{array}{l}\text { Case-control } \\
\text { study }\end{array}$ & 89 & 36 & $\begin{array}{l}\text { CAM-18, CAM-ICU19, and } \\
\text { validated chart review }\end{array}$ \\
\hline $\begin{array}{l}\text { Radcliff et al. } \\
2016[41]\end{array}$ & $\begin{array}{l}\text { The United } \\
\text { States }\end{array}$ & 2010-2012 & Cervical spine surgery & 72.3 & $\begin{array}{l}\text { Retrospective } \\
\text { database study }\end{array}$ & 2792 & 157 & NA \\
\hline $\begin{array}{l}\text { Elsamadicy et al. } \\
2017 \text { [24] }\end{array}$ & $\begin{array}{l}\text { The United } \\
\text { States }\end{array}$ & 2005-2015 & $\begin{array}{l}\text { Spine deformity } \\
\text { surgery }\end{array}$ & 61.4 & $\begin{array}{l}\text { Retrospective } \\
\text { cohort study }\end{array}$ & 923 & 66 & DSM-V criteria \\
\hline $\begin{array}{l}\text { Jiang et al. } 2017 \\
\text { [29] }\end{array}$ & China & 2010-2015 & Mixed spine surgery & 65.1 & Cross-sectional & 451 & 42 & $\begin{array}{l}\text { Clinical Dementia Rating and } \\
\text { Global Deterioration Scale }\end{array}$ \\
\hline $\begin{array}{l}\text { Kobayashi } \\
\text { et al. } \\
2017 \text { [35] }\end{array}$ & Japan & NA & Mixed spine surgery & $\begin{array}{l}\text { Aged } 80 \\
\text { years or } \\
\text { older }\end{array}$ & $\begin{array}{l}\text { Retrospective } \\
\text { database } \\
\text { analysis }\end{array}$ & 262 & 15 & NA \\
\hline $\begin{array}{l}\text { Soh et al. } \\
2017 \text { [43] }\end{array}$ & Korea & 2014-2015 & Mixed spine surgery & $\begin{array}{l}\text { Aged } 73 \\
\text { years or } \\
\text { older }\end{array}$ & $\begin{array}{l}\text { Prospective } \\
\text { observational } \\
\text { study }\end{array}$ & 109 & 9 & ICDSC and CAM-ICU \\
\hline $\begin{array}{l}\text { Adogwa } \\
\text { et al. } \\
2018 \text { [18] }\end{array}$ & $\begin{array}{l}\text { The United } \\
\text { States }\end{array}$ & NA & $\begin{array}{l}\text { Degenerative scoliosis } \\
\text { surgery }\end{array}$ & $\begin{array}{l}\text { Aged } 65 \\
\text { years or } \\
\text { older }\end{array}$ & $\begin{array}{l}\text { Retrospective } \\
\text { cohort study }\end{array}$ & 82 & 22 & CAM \\
\hline $\begin{array}{l}\text { Kim et al. } \\
2018 \text { [32] }\end{array}$ & Korea & 2015-2016 & Mixed spine surgery & 71.7 & $\begin{array}{l}\text { Prospective } \\
\text { cohort study }\end{array}$ & 104 & 15 & CAM \\
\hline $\begin{array}{l}\text { Kobayashi } \\
\text { et al. } \\
2018 \text { [35] }\end{array}$ & Japan & 2008-2013 & Mixed spine surgery & 91.3 & $\begin{array}{l}\text { Prospective } \\
\text { database }\end{array}$ & 35 & 11 & NA \\
\hline $\begin{array}{l}\text { Morino et al. } \\
2018 \text { [38] }\end{array}$ & Japan & 2012-2014 & Mixed spine surgery & 64.2 & $\begin{array}{l}\text { Retrospective } \\
\text { cohort study }\end{array}$ & 532 & 59 & DSM-IV \\
\hline $\begin{array}{l}\text { Susano et al. } \\
2018 \text { [44] }\end{array}$ & $\begin{array}{l}\text { The United } \\
\text { States }\end{array}$ & 2015-2017 & Mixed spine surgery & 73.6 & $\begin{array}{l}\text { Case-control } \\
\text { study }\end{array}$ & 716 & 127 & NA \\
\hline
\end{tabular}


Table 1 Characteristics of included studies (Continued)

\begin{tabular}{|c|c|c|c|c|c|c|c|c|}
\hline Author ", year & Country & Year of survey & Type of operation & $\begin{array}{l}\text { Age } \\
\text { (mean, y) }\end{array}$ & Study design & $\begin{array}{l}\text { Sample } \\
\text { size }\end{array}$ & $\begin{array}{l}n \\
\text { (delirium) }\end{array}$ & Delirium assessment \\
\hline $\begin{array}{l}\text { Cui et al. } \\
2019 \text { [22] }\end{array}$ & China & $2016-2018$ & Mixed spine surgery & 70.2 & $\begin{array}{l}\text { Case-control } \\
\text { study }\end{array}$ & 436 & 112 & CAM \\
\hline $\begin{array}{l}\text { Elsamadicy } \\
\text { et al. } 2019 \text { [25] }\end{array}$ & $\begin{array}{l}\text { The United } \\
\text { States }\end{array}$ & 2010-2015 & Mixed spine surgery & 54.7 & $\begin{array}{l}\text { Retrospective } \\
\text { cohort study }\end{array}$ & 138 & 15 & CAM \\
\hline $\begin{array}{l}\text { Kin et al. } \\
2019 \text { [33] }\end{array}$ & Japan & 2014-2018 & $\begin{array}{l}\text { Surgery for cervical } \\
\text { spondylotic } \\
\text { myelopathy }\end{array}$ & 69.6 & $\begin{array}{l}\text { Retrospective } \\
\text { cohort study }\end{array}$ & 67 & 10 & CAM \\
\hline $\begin{array}{l}\text { Oe et al. } \\
2019 \text { [39] }\end{array}$ & Japan & 2010-2017 & $\begin{array}{l}\text { Spinal deformity } \\
\text { surgery }\end{array}$ & 65.8 & $\begin{array}{l}\text { Retrospective } \\
\text { cohort study }\end{array}$ & 319 & 30 & CAM \\
\hline $\begin{array}{l}\text { Pan et al. } \\
2019 \text { [40] }\end{array}$ & Korea & $2015-2016$ & Lumbar spine surgery & 71.4 & $\begin{array}{l}\text { Retrospective } \\
\text { cohort study }\end{array}$ & 83 & 12 & CAM \\
\hline
\end{tabular}

NA no available, DOS delirium observation screening, CAM Confusion Assessment Method, DSM diagnostic and statistical manual of mental disorders

Table 2 The quality assessment according to the Newcastle Ottawa Scale of each study

\begin{tabular}{|c|c|c|c|c|}
\hline Study & Selection & Comparability & Exposure & Total score \\
\hline Kawaguchi et al. [30] & 3 & 2 & 2 & 7 \\
\hline Cho et al. [21] & 3 & 2 & 2 & 7 \\
\hline Gao et al. [27] & 3 & 2 & 3 & 8 \\
\hline Ushida et al. [45] & 2 & 2 & 3 & 7 \\
\hline Lee and Park [36] & 3 & 2 & 2 & 7 \\
\hline Kelly et al. [31] & 2 & 2 & 2 & 6 \\
\hline Li et al. [37] & 3 & 2 & 2 & 7 \\
\hline Bollen et al. [19] & 2 & 2 & 2 & 6 \\
\hline Fineberg et al. [26] & 3 & 2 & 3 & 8 \\
\hline Dea et al. [23] & 3 & 2 & 2 & 7 \\
\hline Seo et al. [42] & 3 & 2 & 2 & 7 \\
\hline Glennie et al. [28] & 3 & 2 & 3 & 8 \\
\hline Brown et al. [20] & 3 & 2 & 3 & 8 \\
\hline Radcliff et al. [41] & 3 & 2 & 3 & 8 \\
\hline Elsamadicy et al. [24] & 4 & 2 & 3 & 9 \\
\hline Jiang et al. [29] & 3 & 2 & 2 & 7 \\
\hline Kobayashi et al. [35] & 3 & 2 & 3 & 8 \\
\hline Soh et al. [43] & 3 & 2 & 3 & 8 \\
\hline Adogwa et al. [18] & 3 & 2 & 2 & 7 \\
\hline Kim et al. [32] & 3 & 2 & 3 & 8 \\
\hline Kobayashi et al. [35] & 2 & 2 & 2 & 6 \\
\hline Morino et al. [38] & 4 & 2 & 3 & 9 \\
\hline Susano et al. [44] & 3 & 2 & 3 & 8 \\
\hline Cui et al. [22] & 3 & 2 & 3 & 8 \\
\hline Elsamadicy et al. [25] & 4 & 2 & 3 & 9 \\
\hline Kin et al. [33] & 3 & 2 & 2 & 7 \\
\hline Oe et al. [39] & 3 & 2 & 2 & 7 \\
\hline Pan et al. [40] & 4 & 2 & 3 & 9 \\
\hline
\end{tabular}

stable except the pooled results $(7.4 \%$; 95\%CI, $0.069-$ 0.079 ) when excluding the study by Fineberg (Table 3 ). Subgroup analyses indicated that there were significant differences in the incidences of POD among Asia (7.7\%), Europe (5.3\%), and North America (0.8\%), as well as between oncological spinal surgery (5.3\%) and nononcological spinal surgery $(0.9 \%)$. In subgroup analyses stratified by year of survey, the incidence of POD after 2010 (4.1\%) was higher than that before 2010 (0.9\%). Similarly, in stratified analyses by mean age, we observed that the incidence of POD in patients older than 60 years $(8.2 \%)$ was also higher than that in patients younger or equal to 60 years $(0.9 \%)$. In subgroup analysis by study design, the incidence of POD in the subgroup of nondatabase related observational study (8.4\%) was higher than that in the database analysis subgroup (0.8\%). Subgroup analysis based on preoperative disease status showed that the incidence of POD in patients with preoperative cerebrovascular disorders $(8.7 \%)$ was significantly higher than that in patients without preoperative cerebrovascular disorders (0.8\%). When stratified by sample size, the incidence of POD in $\leq 500$ group (8.8\%) was higher than that in $>500$ group $(0.8 \%)$. The subgroup analyses were detailed in Table 4. Furthermore, the meta-regression analyses showed that publication time $(p=0.041)$, but not sample size $(p=0.183)$ and NOS score $(p=0.975)$, was significantly associated with higher POD after spine surgery. The funnel plot seemed to be asymmetric and statistical tests (Egger's test, $p=0.797$ and Begg's test, $p=0.008$; Fig. 3a) also suggested the significant evidence of publication bias. However, the pooled prevalence for POD $(0.9 \%$; 95\%CI, 0.008-0.009) did not change significantly after adding two "missing" studies from the "trim and fill" analysis (Fig. 3b).

\section{Perioperative risk factors for postoperative delirium}

A total of nine risk factors associated with POD after spine surgery were reported on multivariate or adjusted 


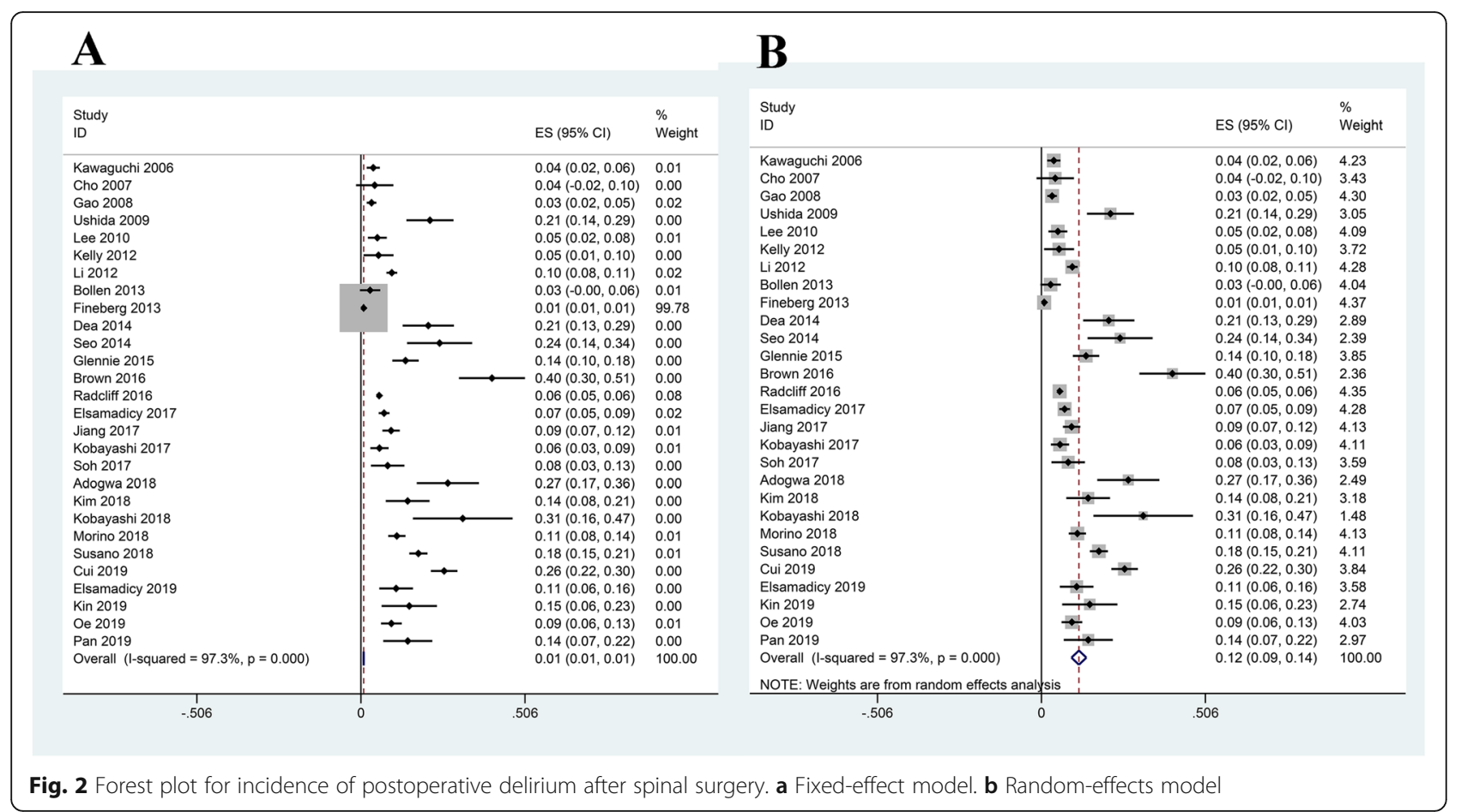

analysis in two or more included studies (Table 5). Of these risk factors, the central nervous system disorder (5 studies; OR 4.73; 95\%CI, 4.30-5.19) was a strong predictor for postoperative delirium, whereas age (10 studies; OR 1.16; 95\%CI, 1.05-2.47; $I^{2}=99.2 \%$ ) and blood loss (four studies; OR 1.10; 95\%CI, 1.01-1.20; $I^{2}=$ 93.3\%) were weaker predictors.

\section{Discussion}

The current study shows that the POD was a serious complication after spine surgery with pooled prevalence ranging from 0.83 to $0.88 \%$. The central nervous system disorder was a strong predictor, whereas age and blood loss were the weaker predictors for POD after spine surgery.

In the present meta-analysis, a total of 28 studies were identified to evaluate the incidence of POD following spinal surgery. The overall pooled prevalence of POD was $0.85 \%$ with substantial heterogeneity. We found that the incidence of POD in elder patients $(>60)$ was higher than that in younger patients $(\leq 60)$, suggesting that older age might be a risk factor for POD after spinal surgery. In accordance with these, our data synthesis analysis revealed that older age was a significant risk factor for POD after spinal surgery (OR 1.16, 95\%CI 1.052.47). In general, the elderly patients usually have poor general health status, more physical and psychological problems, and decreased functioning; all of which might contribute to the occurrence of POD after spinal surgery
[46, 47]. Meta-regression analyses also found that publication time was significantly associated with higher POD prevalence. A possible interpretation was that the global aging population trend in these years may be an important contributor to the increased prevalence of POD accompanied with years [48]. Subgroup analysis also revealed that the pooled prevalence of POD in patients with preoperative cerebrovascular disorders was approximately two times higher than those without preoperative cerebrovascular disorders. Additionally, we found that the central nervous system disorder was identified as a strong predictor for POD after spine surgery as well. Actually, numerous previous studies have demonstrated that some cerebrovascular disorders including Alzheimer's disease and dementia were associated with a high risk of delirium, which may help to explain our findings [49-52]. Our study also found that the male gender may be a significant risk factor for POD after spine surgery. A possible reason was that cerebrovascular disorders have a high prevalence among men versus women [53-55], which may lead to a high risk of POD after spine surgery. Furthermore, we identified that hemoglobin $<100 \mathrm{~g} / \mathrm{L}$, blood loss, and blood transfusion were potential predictors for POD. An important reason for this was that perioperative oxygen insufficiency of the central nervous system facilitated the development of POD after spine surgery. Other risk factors, such as operative time, MMSE score, and ASA score were possible risk factors for POD, although the pooled results 
Table 3 Sensitivity analyses through removing individual studies each time

\begin{tabular}{llll}
\hline Study omitted & Estimate & LCl & UCl \\
\hline Kawaguchi et al. [30] & 0.00853848 & 0.00830359 & 0.00877338 \\
Cho et al. [21] & 0.00854187 & 0.00830699 & 0.00877675 \\
Gao et al. [27] & 0.0085364 & 0.00830149 & 0.00877131 \\
Ushida et al. [45] & 0.0085403 & 0.00830541 & 0.00877518 \\
Lee and Park [36] & 0.0085397 & 0.00830482 & 0.00877459 \\
Kelly et al. [31] & 0.00854126 & 0.00830637 & 0.00877614 \\
Li et al. [37] & 0.00852485 & 0.00828995 & 0.00875976 \\
Bollen et al. [19] & 0.00854134 & 0.00830645 & 0.00877623 \\
Fineberg et al. [26] & 0.07398792 & 0.06900876 & 0.07896708 \\
Dea et al. [23] & 0.00854068 & 0.0083058 & 0.00877556 \\
Seo et al. [42] & 0.00854115 & 0.00830627 & 0.00877603 \\
Glennie et al. [28] & 0.00853812 & 0.00830324 & 0.00877301 \\
Brown et al. [20] & 0.00854033 & 0.00830545 & 0.00877521 \\
Radcliff et al. [41] & 0.00850637 & 0.00827141 & 0.00874134 \\
Elsamadicy et al. [24] & 0.00852986 & 0.00829496 & 0.00876476 \\
Jiang et al. [29] & 0.00853595 & 0.00830106 & 0.00877083 \\
Kobayashi et al. [35] & 0.00853904 & 0.00830415 & 0.00877393 \\
Soh et al. [43] & 0.0085409 & 0.00830602 & 0.00877579 \\
Adogwa et al. [18] & 0.00854088 & 0.00830599 & 0.00877576 \\
Kim et al. [32] & 0.00854079 & 0.00830591 & 0.00877567 \\
Kobayashi et al. [35] & 0.00854172 & 0.00830684 & 0.0087766 \\
Morino et al. [38] & 0.0085345 & 0.00829961 & 0.00876939 \\
Susano et al. [44] & 0.00853053 & 0.00829565 & 0.00876542 \\
Cui et al. [22] & 0.00853429 & 0.0082994 & 0.00876917 \\
Elsamadicy et al. [25] & 0.00854038 & 0.0083055 & 0.00877527 \\
Kin et al. [33] & 0.00854137 & 0.00830649 & 0.00877625 \\
Oe et al. [39] & 0.00853784 & 0.00830295 & 0.00877272 \\
Pan et al. [40] & 0.00854112 & 0.00830624 & 0.008776 \\
\hline
\end{tabular}

$\mathrm{LCl}$ low confidence interval, $\mathrm{UCl}$ upper confidence interval

were no statistical significant. Taken together, perioperative management focused on these aforementioned risk factors may reduce the risk of POD after spine surgery.

The current study also existed many limitations. Firstly, in the current study, the results of pooled analysis based on random-effects model and fixed-effect model are very different from each other owing to substantial heterogeneity across included studies. According to the Cochrane Handbook (9.5.4), the random-effects estimate and its confidence interval address the question "what is the average effect?" while the fixed-effect estimate and its confidence interval address the question "what is the best estimate of the effect?" Therefore, we just chose the fixed-effect estimate and its confidence interval, but not the random-effects estimate, which may
Table 4 Subgroup analysis of delirium after spine surgery

\begin{tabular}{|c|c|c|c|}
\hline Outcomes & $\begin{array}{l}\text { Number } \\
\text { of trials }\end{array}$ & $\begin{array}{l}\text { Pooled } \\
\text { prevalence } \\
\text { with } 95 \% \mathrm{Cl}\end{array}$ & $R^{2}(\%)$ \\
\hline Primary analysis & 28 & $0.009(0.008-0.009)$ & 97.3 \\
\hline \multicolumn{4}{|l|}{$\begin{array}{l}\text { Subgroup analyses } \\
\text { based on the region }\end{array}$} \\
\hline Asia & 17 & $0.077(0.069-0.084)$ & 91.3 \\
\hline Europe & 2 & $0.053(0.024-0.082)$ & 94.1 \\
\hline North America & 9 & $0.008(0.008-0.009)$ & 98.3 \\
\hline \multicolumn{4}{|l|}{$\begin{array}{l}\text { Subgroup analyses based } \\
\text { on the year of survey }\end{array}$} \\
\hline Before 2010 & 5 & $0.041(0.030-0.052)$ & 82.8 \\
\hline After 2010 & 23 & $0.009(0.008-0.009)$ & 97.7 \\
\hline \multicolumn{4}{|l|}{$\begin{array}{l}\text { Subgroup analyses based } \\
\text { on the sample size }\end{array}$} \\
\hline$>500$ & 7 & $0.008(0.008-0.009)$ & 98.8 \\
\hline$\leq 500$ & 21 & $0.088(0.079-0.097)$ & 90.9 \\
\hline \multicolumn{4}{|l|}{$\begin{array}{l}\text { Subgroup analyses based } \\
\text { on the type of operation }\end{array}$} \\
\hline Oncological spine surgery & 2 & $0.053(0.024,0.082)$ & 94.1 \\
\hline Non-oncological spine surgery & 26 & $0.009(0.008-0.009)$ & 97.4 \\
\hline \multicolumn{4}{|l|}{$\begin{array}{l}\text { Subgroup analyses based } \\
\text { on the mean age of candidate } \\
\text { patients }\end{array}$} \\
\hline$>60$ & 22 & $0.082(0.077-0.088)$ & 92.3 \\
\hline$\leq 60$ & 6 & $0.009(0.008-0.009)$ & 93.2 \\
\hline \multicolumn{4}{|l|}{$\begin{array}{l}\text { Subgroup analyses based on } \\
\text { the study design }\end{array}$} \\
\hline Database analysis & 4 & $0.008(0.008-0.009)$ & 98 \\
\hline $\begin{array}{l}\text { Non-database related } \\
\text { observational study }\end{array}$ & 24 & $0.084(0.078-0.09)$ & 92.4 \\
\hline \multicolumn{4}{|l|}{$\begin{array}{l}\text { Subgroup analyses based on } \\
\text { the preoperative disease status }\end{array}$} \\
\hline $\begin{array}{l}\text { Patients with not preoperative } \\
\text { cerebrovascular disorders }\end{array}$ & 28 & $0.008(0.008-0.009)$ & 97.2 \\
\hline $\begin{array}{l}\text { Patients with preoperative } \\
\text { cerebrovascular disorders }\end{array}$ & 3 & $0.087(0.079-0.093)$ & 97.4 \\
\hline
\end{tabular}

more truthfully reflect the pooled prevalence of POD in spine surgery. The choice may be inappropriate, but can show the authentic pooled prevalence of POD in spine surgery.

Our study indicated that the overall pooled prevalence of POD was approximately $0.85 \%$ with substantial heterogeneity. Meta-regression analyses indicated that the year of publication was a significant contributor to statistical heterogeneity. Moreover, we performed subgroup analyses based on the year of survey and found that the incidence of POD after 2010 was lower than those before 2010. However, the statistical heterogeneity within subgroups was still substantial, so there may exist other 

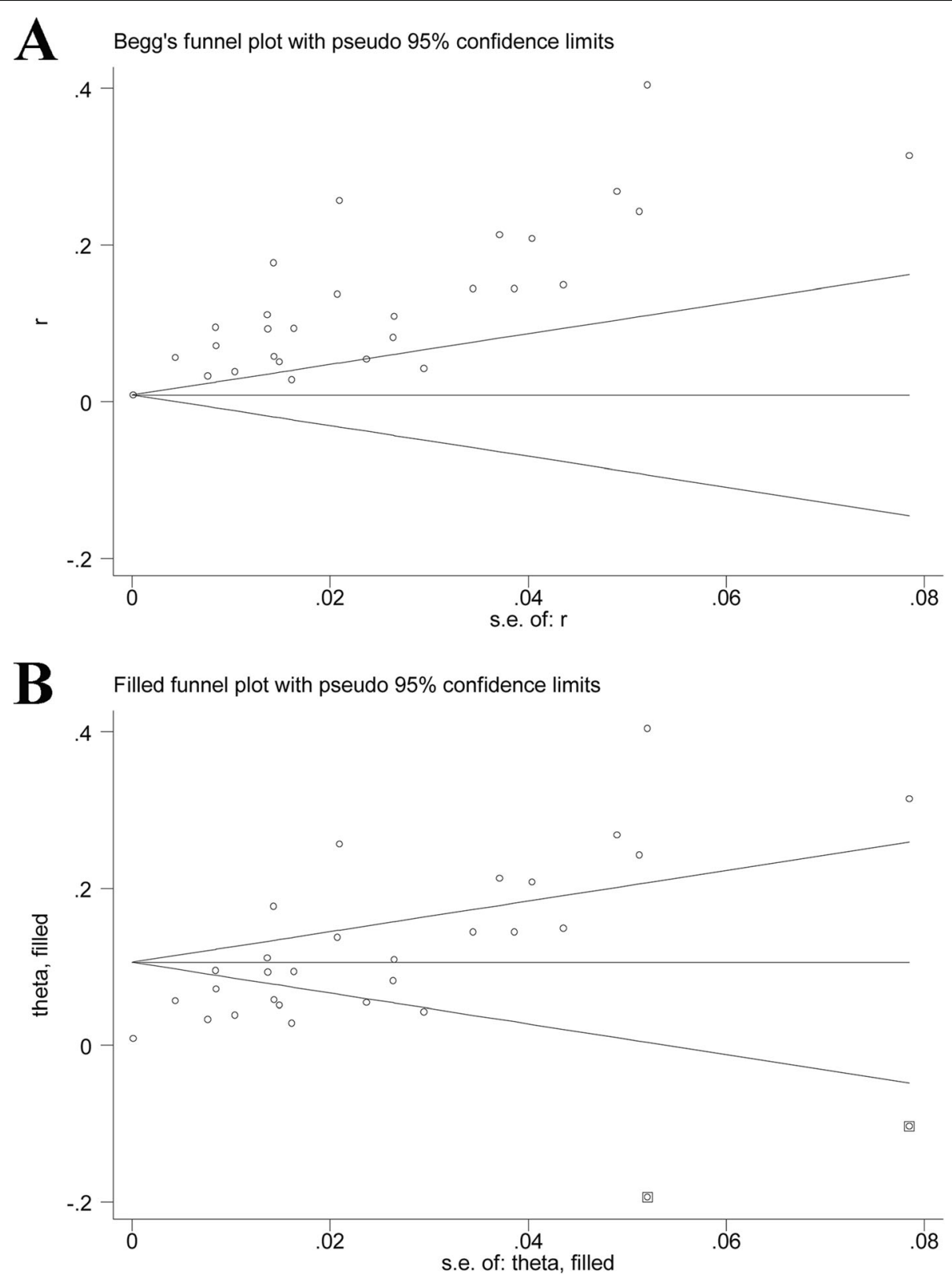

Fig. 3 Funnel plot of postoperative delirium after spinal surgery (Egger's test, $p=0.797$ and Begg's test, $p=0.008$ ). a Adjusted funnel plot of postoperative delirium after spinal surgery after adding two "missing" studies from the "trim and fill" analysis (b)

significant sources of heterogeneity. Understandably, the statistical heterogeneity may not attribute to individual factors, such as publication time, but many clinical and methodological difference factors across included studies including demographic characteristics, type of operation, country, study design, and the definition or duration of POD. Secondly, most of the included patients in the current meta-analysis were from Steven J. Fienberg's study which accounts for $98.25 \%$ of the total patients. Therefore, the results of the study may be potentially skewed in favor of statistics reported by Fineberg.
Additionally, we also performed the sensitivity analyses by sequentially excluding single study and subgroup analyses to explore the robustness and creditability of our overall pooled effect. We found that there is a high possibility that our pooled result was skewed in favor of the statistics reported by Fineberg. Of course, we cannot totally exclude the bias risk since all the included studies were retrospective studies and unavoidable heterogeneity. Thus, further homogeneous and prospective studies should be warranted to elucidate the prevalence of POD following spine surgery. Thirdly, some risk factors were 
Table 5 Meta-analysis of risk factors for postoperative delirium in spine surgery

\begin{tabular}{llll}
\hline Outcomes & $\begin{array}{l}\text { Number of } \\
\text { trials }\end{array}$ & OR $(95 \% \mathrm{Cl})$ & $P^{2}(\%)$ \\
\hline Age & 10 & $1.61(1.05-2.47)$ & 99.2 \\
Sex (men) & 4 & $1.11(0.36-3.46)$ & 60.3 \\
Hemoglobin < $100 \mathrm{~g} / \mathrm{L}$ & 2 & $0.61(0.19-1.97)$ & 76 \\
$\begin{array}{l}\text { Central nervous system } \\
\text { disorder }\end{array}$ & 5 & $4.73(4.30-5.19)$ & 0 \\
Blood loss & 5 & $1.10(1.01-1.20)$ & 93.3 \\
Blood transfusion & 2 & $2.57(0.95-6.93)$ & 0 \\
Operative time & 2 & $0.99(0.97-1.01)$ & 0 \\
MMSE score & 3 & $1.03(0.62-1.69)$ & 84.6 \\
ASA score & 2 & $4.25(0.86-20.93)$ & 92 \\
\hline
\end{tabular}

MMSE Mini-Mental State Examination, ASA American Society of Anesthesiologists

reported in limited included studies, but the pooled estimate based on limited studies may bias the authenticity. The pooled analysis based on two studies found that the ASA score was associated with more than four-time risk of POD after spine surgery, but with no statistical significance. Actually, many studies revealed ASA score was a significant predictor for POD [56-59]. Moreover, there are other factors like electrolyte imbalance and general condition of the patient before surgery which were not identified in the current meta-analysis, but may also be potential risk factors for POD. However, these factors were reported in the limited studies, so we did not include them in this meta-analysis for the pooled estimate, considering that the limited studies may bias the authenticity of our pooled analysis. Therefore, our results in this meta-analysis may be too conservative and should be interpreted cautiously. Meanwhile, many other potential risk factors for POD should be further assessed in future studies. Finally, the funnel plot and statistical tests suggested that the current meta-analysis may exist publication bias, regardless of the fact that we have performed a systematic literature search. However, the pooled prevalence for POD basically remained stable after adding two "missing" studies, which further supported the reliability of the pooled effect.

To sum up, our study indicated that the pooled POD after spinal surgery was approximately $0.85 \%$. The central nervous system disorder, age, and blood loss were potential risk factors for POD. Further studies with more homogeneous clinical parameters should be warranted to illuminate the prevalence and risk factors of POD after spine surgery.

\section{Abbreviations}

Cl: Confidence interval; OR: Odds ratio; POD: Postoperative delirium

\section{Acknowledgements}

We thank the authors of the included studies for their help.

\section{Authors' contributions}

Hua Gao designed this meta-analysis. Hua Gao and Hui-Juan Ma extracted data. Ying-Jia Li performed the statistical analysis. Hua Gao, Ci Yin, and Zheng

Li wrote this manuscript. The authors read and approved the final manuscript

Funding

No fund support

\section{Availability of data and materials}

All data are fully available without restriction.

Ethics approval and consent to participate

This article does not contain any studies with human participants or animals performed by any of the authors

Consent for publication

Not applicable

\section{Competing interests}

The authors declare that they have no competing interests.

\section{Author details}

${ }^{1}$ Department of Outpatient, Lanzhou University Second Hospital, Lanzhou University Second Clinical Medical College, Lanzhou 730030, China.

${ }^{2}$ Operating Theater, Lanzhou University Second Hospital, Lanzhou University Second Clinical Medical College, Lanzhou 730030, China. ${ }^{3}$ Department of

Thoracic Surgery, Lanzhou University Second Hospital, Lanzhou University Second Clinical Medical College, Lanzhou 730030, China.

Received: 4 January 2020 Accepted: 27 March 2020

Published online: 09 April 2020

\section{References}

1. Inouye SK, Westendorp RG, Saczynski JS. Delirium in elderly people. Lancet. 2014;383:911-22.

2. Bryczkowski SB, Lopreiato MC, Yonclas PP, et al. Delirium prevention program in the surgical intensive care unit improved the outcomes of older adults. J Surg Res. 2014;190:280-8.

3. Oh ES, Fong TG, Hshieh TT, et al. Delirium in older persons: advances in diagnosis and treatment. JAMA. 2017;318:1161-74.

4. Bhattacharya B, Maung A, Barre $K$, et al. Postoperative delirium is associated with increased intensive care unit and hospital length of stays after liver transplantation. J Surg Res. 2017:207:223-8.

5. Ashley KE, Hillegass WB. Costs of postoperative delirium with transcatheter aortic valve replacement: improved yet still present. Catheter Cardiovasc Interv. 2019;93:1137.

6. Daiello LA, Racine AM, Yun Gou R, et al. Postoperative delirium and postoperative cognitive dysfunction: overlap and divergence. Anesthesiology 2019.

7. Vlisides $P$, and Avidan M. Recent advances in preventing and managing postoperative delirium. F1000Res. 2019; 8.

8. Shi C, Yang C, Gao R. et al. Risk factors for delirium after spinal surgery: a meta-analysis. .

9. Liao Y, Flaherty JH, Yue J, et al. The incidence of delirium after cardiac surgery in the elderly: protocol for a systematic review and meta-analysis. BMJ Open. 2017;7:e014726.

10. Texakalidis P, Tzoumas A, Giannopoulos S, et al. Risk factors for restenosis following carotid revascularization: a meta-analysis of hazard ratios. World Neurosurg. 2019

11. Gul W, Fuller HR, Wright $H$, et al. A systematic review and meta-analysis of the effectiveness of surgical decompression in treating patients with malignant middle cerebral artery infarction. World Neurosurg. 2018;120: e902-20.

12. Hong EP, Kim BJ, Jeon JP, et al. Association of endothelin receptor type A with intracranial aneurysm in 20,609 East Asians: an updated meta-analysis. World Neurosurg. 2019. 
13. Kim BS, Kim KH, Lee MH, et al. Stereotactic radiosurgery for brainstem cavernous malformations: an updated systematic review and meta-analysis World Neurosurg. 2019.

14. Lu VM, Wahood W, Akinduro OO, et al. Four independent predictors of postoperative seizures after meningioma surgery: a meta-analysis. World Neurosurg. 2019

15. Begg CB, Mazumdar M. Operating characteristics of a rank correlation test for publication bias. Biometrics. 1994;50:1088-101.

16. Weng $S$, Wang W, Wei $Q$, et al. Effect of tranexamic acid in patients with traumatic brain injury: a systematic review and meta-analysis. World Neurosurg. 2019;123:128-35.

17. Zhang Y, Liu W, Xu J. Prognostic utility and clinical significance of lysyl oxidase-like 2 protein expression in digestive system cancers. J Cell Physiol. 2019.

18. Adogwa O, Elsamadicy AA, Vuong VD, et al. Association between baseline cognitive impairment and postoperative delirium in elderly patients undergoing surgery for adult spinal deformity. Journal of neurosurgery. Spine. 2018;28:103-8.

19. Bollen L, de Ruiter Gc Fau - Pondaag W, Pondaag W Fau - Arts MP, et al. Risk factors for survival of 106 surgically treated patients with symptomatic spinal epidural metastases.

20. Brown $\mathrm{CH}$, LaFlam A, Max L, et al. Delirium after spine surgery in older adults: incidence, risk factors, and outcomes. Journal of the American Geriatrics Society. 2016;64:2101-8.

21. Cho KJ, Suk SI, Park SR, et al. Complications in posterior fusion and instrumentation for degenerative lumbar scoliosis. Spine. 2007;32:2232-7.

22. Cui $X P$, Jing $Z Z$, Song JF, et al. A retrospective study on risk factors associated with postoperative delirium in elderly patients with spinal operation. Zhongguo gu shang = China journal of orthopaedics and traumatology. 2019; 32: 549-554.

23. Dea N, Versteeg A, Fisher C, et al. Adverse events in emergency oncological spine surgery: a prospective analysis. Journal of neurosurgery. Spine. 2014;21: 698-703.

24. Elsamadicy AA, Adogwa O, Lydon E, et al. Depression as an independent predictor of postoperative delirium in spine deformity patients undergoing elective spine surgery. Journal of neurosurgery. Spine. 2017;27:209-14.

25. Elsamadicy AA, Charalambous LT, Sergesketter AR, et al. Intraoperative ketamine may increase risk of post-operative delirium after complex spinal fusion for adult deformity correction. Journal of spine surgery (Hong Kong). 2019; 5: 79-87.

26. Fineberg SJ. Nandyala Sv Fau - Marquez-Lara A, Marquez-Lara A Fau Oglesby $\mathrm{M}$, et al. Incidence and risk factors for postoperative delirium after lumbar spine surgery.

27. Gao R, Yang ZZ, Li M, et al. Probable risk factors for postoperative delirium in patients undergoing spinal surgery. European Spine Journal. 2008;17: 1531-7.

28. Glennie RA, Ailon T, Yang K, et al. Incidence, impact, and risk factors of adverse events in thoracic and lumbar spine fractures: an ambispective cohort analysis of 390 patients. The spine journal : official journal of the North American Spine Society. 2015;15:629-37.

29. Jiang $X$, Chen D, Lou Y, et al. Risk factors for postoperative delirium after spine surgery in middle- and old-aged patients. Aging clinical and experimental research. 2017;29:1039-44.

30. Kawaguchi $Y$, Kanamori $M$, Ishihara $H$, et al. Postoperative delirium in spine surgery. The spine journal : official journal of the North American Spine Society. 2006;6:164-9.

31. Kelly A, Batke JN, Boyd M, et al. Prospective analysis of adverse events in surgical treatment of degenerative spondylolisthesis. Spine Journal. 2012;12: 108S-9S.

32. Kim KH, Kang SY, Shin DA, et al. Parkinson's disease-related non-motor features as risk factors for post-operative delirium in spinal surgery. PLoS ONE. 2018;13.

33. Kin K, Yasuhara T, Tomita Y, et al. SF-36 scores predict postoperative delirium after surgery for cervical spondylotic myelopathy. Journal of neurosurgery. Spine. 2019:1-6.

34. Kobayashi K, Imagama S, Ando K, et al. Risk factors for delirium after spine surgery in extremely elderly patients aged 80 years or older and review of the literature: Japan Association of Spine Surgeons with ambition multicenter study. Global Spine Journal. 2017;7:560-6.
35. Kobayashi K, Imagama S, Sato K, et al. Postoperative complications associated with spine surgery in patients older than 90 years: a multicenter retrospective study. Global Spine J. 2018;8:887-91.

36. Lee JK, Park YS. Delirium after spinal surgery in Korean population. Spine. 2010;35:1729-32.

37. $\mathrm{Li} H, \mathrm{Li} C D, Y i X D$, et al. Analysis of risk factors for delirium in the elderly patients after spinal operation. Beijing da xue xue bao. Yi xue ban = Journal of Peking University. Health sciences. 2012; 44: 847-850.

38. Morino T, Hino M, Yamaoka $\mathrm{S}$, et al. Risk factors for delirium after spine surgery: an age-matched analysis. Asian spine journal. 2018;12:703-9.

39. Oe $S$, Togawa D, Yamato $Y$, et al. Preoperative age and prognostic nutritional index are useful factors for evaluating postoperative delirium among patients with adult spinal deformity. Spine. 2019;44:472-8.

40. Pan Z, Huang $K$, Huang $W$, et al. The risk factors associated with delirium after lumbar spine surgery in elderly patients. Quantitative imaging in medicine and surgery. 2019;9:700-10.

41. Radcliff K, Ong KL, Lovald S, et al. Cervical spine surgery complications and risks in the elderly. Spine. 2017;42:E347-e354.

42. Seo JS, Park SW, Lee YS, et al. Risk factors for delirium after spine surgery in elderly patients. Journal of Korean Neurosurgical Society. 2014;56:28-33.

43. Soh S, Shim JK, Song JW, et al. Postoperative delirium in elderly patients undergoing major spinal surgery: role of cerebral oximetry. J Neurosurg Anesthesiol. 2017;29:426-32.

44. Susano MJ, Scheetz SD, Grasfield RH, et al. Retrospective analysis of perioperative variables associated with postoperative delirium and other adverse outcomes in older patients after spine surgery. J Neurosurg Anesthesiol. 2018.

45. Ushida T, Yokoyama T, Kishida Y, et al. Incidence and risk factors of postoperative delirium in cervical spine surgery. Spine. 2009;34:2500-4

46. Tanaka M, Tani N, Maruo T, et al. Risk factors for postoperative delirium after deep brain stimulation surgery for parkinson disease. World Neurosurg. 2018; 114:e518-23.

47. Wang XQ, Zhuang HX. Zhang LX, et al. World Neurosurg: Nomogram for predicting postoperative delirium after deep brain stimulation surgery for Parkinson's disease; 2019.

48. Fukuoka H, Afshari NA. The impact of age-related cataract on measures of frailty in an aging global population. Curr Opin Ophthalmol. 2017;28:93-7.

49. Fong TG, Davis D, Growdon ME, et al. The interface between delirium and dementia in elderly adults. Lancet Neurol. 2015;14:823-32.

50. FitzGerald JM, Perera G, Chang-Tave A, et al. The incidence of recorded delirium episodes before and after dementia diagnosis: differences between dementia with lewy bodies and Alzheimer's disease. J Am Med Dir Assoc. 2019;20:604-9.

51. Hov KR, Bolstad N, Idland AV, et al. Cerebrospinal fluid S100B and Alzheimer's disease biomarkers in hip fracture patients with delirium. Dement Geriatr Cogn Dis Extra. 2017;7:374-85.

52. Racine AM, Fong TG, Travison TG, et al. Alzheimer's-related cortical atrophy is associated with postoperative delirium severity in persons without dementia. Neurobiol Aging. 2017;59:55-63.

53. de Havenon A, Meyer C, McNally JS, et al. Subclinical cerebrovascular disease: epidemiology and treatment. Curr Atheroscler Rep. 2019;21:39.

54. McKetton L, Cohn M, Tang-Wai DF, et al. Cerebrovascular resistance in healthy aging and mild cognitive impairment. Front Aging Neurosci. 2019;11:79.

55. Tong X, Yang Q, Ritchey MD, et al. The burden of cerebrovascular disease in the United States. Prev Chronic Dis. 2019;16:E52.

56. Raats JW, Steunenberg SL, de Lange DC, et al. Risk factors of post-operative delirium after elective vascular surgery in the elderly: a systematic review. Int J Surg. 2016;35:1-6.

57. Raats JW, van Eijsden WA, Crolla RM, et al. Risk factors and outcomes for postoperative delirium after major surgery in elderly patients. PLoS One. 2015;10:e0136071.

58. Tei M, Ikeda M, Haraguchi N, et al. Risk factors for postoperative delirium in elderly patients with colorectal cancer. Surg Endosc. 2010;24:2135-9.

59. Tei M, Wakasugi M, Kishi K, et al. Incidence and risk factors of postoperative delirium in elderly patients who underwent laparoscopic surgery for colorectal cancer. Int J Colorectal Dis. 2016;31:67-73.

\section{Publisher's Note}

Springer Nature remains neutral with regard to jurisdictional claims in published maps and institutional affiliations. 